\title{
SPOTLIGHT
}

\section{Articles of Significant Interest Selected from This Issue by the Editors}

\section{Vaccinia Virus Activation of CCR5 Confers a Permissive Phenotype for Infection}

Vaccinia virus enters both permissive and nonpermissive cells. The precise events that allow viral replication and thereby confer permissiveness are ill-defined. Rahbar et al. (p. 7245-7259) provide intriguing evidence for a role of the chemokine receptor, CCR5, in enabling the permissive phenotype. Virus-mediated CCR5 activation leads to a cascade of tyrosine phosphorylation signaling events that are necessary for late vaccinia virus gene expression. These findings implicate host cell receptor activation as a critical determinant of permissiveness for vaccinia virus infection but not viral entry.

\section{Murine Transgenic Model Exposes New Function for LMP2A in B-Cell Antibody Production}

Previous studies indicate that expression of Epstein-Barr virus (EBV) latent membrane protein 2A (LMP2A) in resting murine B cells enhances B-cell receptor (BCR)-derived signals. Swanson-Mungerson et al. (p. 6764-6770) now demonstrate that murine B cells expressing both LMP2A and BCR produce more antibody after antigen exposure than littermate controls in vitro and in vivo. The data indicate that LMP2A increases antibody production by multiple mechanisms, including increased B-cell survival and plasma cell differentiation. Taken together, these findings suggest that LMP2A enhances B-cell function, which may have pathological consequences, such as production of autoantibodies and heterophile antibodies in EBV-infected persons with infectious mononucleosis.

\section{A Fish Virus Protein Protects Double-Stranded RNA from the Host}

Fish betanodavirus nonstructural protein B2 antagonizes RNA interference in animal cells, facilitating the intracellular accumulation of viral RNA. Fenner et al. (p. 6822-6833) show that B2 protects virus-derived double-stranded RNAs (dsRNAs) that would otherwise serve as substrates for cellular antiviral proteins involved in RNA silencing and RNA editing. Unlike many other viral dsRNA-binding proteins, B2 does not prevent induction of host interferon genes. Knowledge of this betanodavirus defense mechanism opens a new avenue of research for the development of attenuated betanodavirus vaccines.

\section{Differentiation between "Self" and "Nonself" in the Interferon Response}

Bovine viral diarrhea virus (BVDV), a relative of hepatitis C virus, may cause persistent infections in early bovine embryos. Schweizer et al. (p. 6926-6935) report that once infection is established in cultured cells, BVDV is not eliminated by alpha/beta interferon (IFN- $\alpha / \beta)$. In contrast, such cells are protected by IFN against infection by two unrelated challenge viruses. This intriguing differentiation between "self" and "nonself" in the IFN response at the single cell level may be important for survival during persistent viral infections. Thus, while not terminating persistence of the residing virus, IFN may nevertheless protect against viral newcomers.

\section{Human Immunodeficiency Virus-Specific $\mathrm{CD}^{+}{ }^{+} \mathrm{T}$ Cells Suffer the Consequences of Preferential Infection}

Human immunodeficiency virus (HIV) preferentially infects virus-specific $\mathrm{CD} 4^{+} \mathrm{T}$ cells. However, the biological consequences of this phenomenon are unclear. Brenchley et al. (p. 6801-6809) demonstrate that this preferential infection limits the expansion and differentiation of HIV-specific $\mathrm{CD} 4^{+} \mathrm{T}$ cells in vivo. Furthermore, they show that preferential infection can be averted in the presence of cross-reactive $\mathrm{CD}^{+} \mathrm{T}$ cells specific for coexistent antigens; under these conditions, high frequencies of terminally differentiated HIV-specific $\mathrm{CD} 4^{+} \mathrm{T}$ cells were observed. These results indicate that preferential infection provides a mechanism whereby HIV subverts the specific antiviral immune response and suggest a potential solution to this problem. 


\section{Human Immunodeficiency Virus (HIV) Antibodies Targeting a Variable Epitope Show Cross-Reactivity between HIV Subtypes}

Identification of targets in human immunodeficiency virus (HIV) for humoral immune responses is of critical importance for the development of HIV vaccines. Gorny et al. (p. 6865-6872) generated human monoclonal antibodies by using cells of subjects infected with strains of HIV-1 carrying the envelope proteins of viral subtypes A or B. The antibodies were directed to the third variable region (V3) of the virus envelope glycoprotein. Despite the sequence variation in this region, the antibodies were immunologically cross-reactive between several HIV subtypes. Antibodies from subjects infected with subtype A viruses appeared to have a broader pattern of reactivity than those from subtype B-infected individuals. These data suggest that a monovalent vaccine should preferentially include a V3 region typical of subtype A or other non-B strains. Alternatively, a polyvalent vaccine would benefit from a combination of V3 epitopes from both $\mathrm{B}$ and non-B strains. 